Draft version August 20, 2019

Preprint typeset using $\mathrm{LATE}_{\mathrm{E}} \mathrm{X}$ style emulateapj v. 11/10/09

\title{
TOMOGRAPHIC RECONSTRUCTION OF THE THREE-DIMENSIONAL STRUCTURE OF THE HH30 JET
}

\author{
F. De COLle ${ }^{1}$ \\ Astronomy \& Astrophysics Department, University of California, Santa Cruz, CA 95064, USA \\ C. DEL Burgo ${ }^{2}$ \\ UNINOVA-CA3, Campus da Caparica, Quinta da Torre, Monte de Caparica 2825-149, Caparica, Portugal \\ AND \\ A. C. RAGA ${ }^{3}$ \\ Instituto de Ciencias Nucleares, Universidad Nacional Autónoma de México, Ap.P. 70543, 04510 DF, Mexico \\ (Dated: Received xxx; accepted xxx) \\ Draft version August 20, 2019
}

\begin{abstract}
The physical parameters of Herbig-Haro jets are usually determined from emission line ratios, obtained from spectroscopy or narrow band imaging, assuming that the emitting region is homogeneous along the line of sight. Under the more general hypothesis of axisymmetry, we apply tomographic reconstruction techniques to the analysis of Herbig-Haro jets. We use data of the HH30 jet taken by Hartigan \& Morse (2007) with the Hubble space telescope using the slitless spectroscopy technique. Using a non-parametric Tikhonov regularization technique, we determine the volumetric emission line intensities of the $[\mathrm{S}$ II $] \lambda \lambda 6716,6731,[\mathrm{O} \mathrm{I}] \lambda 6300$ and $[\mathrm{N}$ II] $\lambda 6583$ forbidden emission lines. From our tomographic analysis of the corresponding line ratios, we produce "three-dimensional" images of the physical parameters. The reconstructed density, temperature and ionization fraction present much steeper profiles than those inferred using the assumption of homogeneity. Our technique reveals that the reconstructed jet is much more collimated than the observed one close to the source (a width $\sim 5 \mathrm{AU}$ vs. $\sim 20 \mathrm{AU}$ at a distance of $10 \mathrm{AU}$ from the star), while they have similar widths at larger distances. In addition, our results show a much more fragmented and irregular jet structure than the classical analysis, suggesting that the the ejection history of the jet from the star-disk system has a shorter timescale component $(\sim$ some months) superimposed on a longer, previously observed timescale (of a few years). Finally, we discuss the possible application of the same technique to other stellar jets and planetary nebulae.

Subject headings: ISM: Herbig-Haro objects - ISM: jets and outflows - Techniques: image processing - Methods: data analysis - Stars: pre-main sequence
\end{abstract}

\section{INTRODUCTION}

Herbig-Haro (HH) jets appear as narrow and well collimated ( a few degrees) knotty beams of atomic/ionic and/or molecular gas moving away from the source with supersonic velocities $\left(\sim 100-300 \mathrm{~km} \mathrm{~s}^{-1}\right)$ (e.g. Reipurth \& Bally 2001).

While considerable progress has been obtained in the understanding of the behavior of $\mathrm{HH}$ jets, long standing problems remain unsolved, including the origin of the stellar jets themselves (e.g. Ferreira 1997; Shu et al. 2000), the importance of jets in solving the angular momentum problem (e.g. Hartmann 2009), the origin of knots along stellar jets (e.g. De Colle et al. 2008b), the feedback of jets on the core and the star formation efficiency, and the interaction of jets with the ambient medium eventually leading to the generation of turbulence in molecular clouds (e.g. Carroll et al. 2009). To properly understand these phenomena, it is crucial to accurately determine the physical conditions of the jet plasma.

The proper determination of the electron density $n_{\mathrm{e}}$,

fabio@ucolick.org

cburgo@uninova.pt

raga@nucleares.unam.mx temperature $T_{\mathrm{e}}$, and hydrogen ionization fraction $x_{\mathrm{H}}$ gives strong insights, for instance, on the nature of the heating source of the jet material, on the mechanism that leads to the production of the knots and on the interaction of the outflowing material with the surrounding environment. The determination of the total density $n_{\mathrm{H}}$ $\left(=n_{\mathrm{e}} / x_{\mathrm{H}}\right)$ is important in order to derive the mass flux, and more significantly, to understand the role played by jets in the removal of angular momentum from the star and the circumstellar disk. Furthermore, different jet ejection models predict different dependencies of $n_{\mathrm{H}}$ as a function of the distance from the jet axis (e.g. Shu et al. 1995; Lerv \& Frank 2000).

Two approaches are used to infer the outflow excitation conditions from the observations. The physical parameters can be calculated directly from the observed line ratios (e.g. Bacciotti \& Eislöffel 1999; Nisini et al. 2005; Podio et al. 2006; Hartigan \& Morse 2007, hereafter HM07). Alternatively, the observations can be compared with predictions of emission line intensities from models of different complexity, ranging from "simple" parallel shock models (e. g. Hartigan et al. 1987, Hartigan et al. 1994, Lavalley-Fouquet et al. 2000, Pesenti et al. 2003) to complex multi-species numerical simulations (e.g. Raga et al. 2007). All these studies 
agree that the jet is denser close to the source, with temperatures and ionization fractions which, although peaking at the positions of the knots, tend to decrease for larger distances from the source.

While previous studies have focused on the variation of the physical conditions along the jet axis, recent imaging of resolved jets has been performed (e.g. Bacciotti et al. 2000, 2002; Coffey et al. 2004, 2007; Woitas et al. 2005; Beck et al. 2007, HM07). It has been observed in these studies that the electron and total densities peak on the axis of the jet and decrease away from the axis (within e.g. a factor of 2 in HM07). The temperature and ionization fraction cross-sections are found to be more homogeneous.

We note, however, that the above-mentioned findings were obtained assuming that stellar jets are homogeneous along the line of sight. In a previous paper (De Colle et al. 2008a, hereafter Paper I) we discussed in detail the error in the determination of the physical parameters introduced when assuming a jet as homogeneous along the line of sight. In Paper I we proposed the application of tomographic techniques to determine the volumetric physical parameters, showing an example of the application of a parametric "multi-Gaussian" (Bendinelli 1991) method to reconstruct the cross-section of the HH30 jet at one particular position along the jet. In this paper, we extend the previous results by reconstructing the full two dimensional structure of the HH30 jet by implementing a non-parametric Tikhonov reconstruction technique.

In particular, Section 2 explains in detail the problems related to the inversion of the physical parameters, and the methods employed in the inversion problem. Section 3 presents the application of the reconstruction process to the HH30 jet. Section 4 and 5 present a discussion of the results and our conclusions.

\section{THE DETERMINATION OF THE JET STRUCTURE}

\subsection{General considerations}

We consider a jet with known physical parameters (hereafter, we refer to the jet electron density $n_{\mathrm{e}}$, electron temperature $T_{\mathrm{e}}$ and ionization fraction $x_{\mathrm{H}}$ as the physical parameters of the jet). To determine synthetic emissivities from the physical parameters it is necessary to follow a series of steps (e.g. Dougados et al. 2010):

1. The emission coefficients are calculated, assuming statistical equilibrium and determining the population of each level for each considered atom/ion.

2. The emission coefficients are integrated along the line of sight.

3. The synthetic emissivity are convolved with the seeing (i.e., blurring and scintillation of the jet in the Earth's atmosphere), the response of the detector, the sampling design and the instrumental point spread function (PSF).

The inverse process consists of deriving the emission coefficients from the observed emission lines, and determining the physical parameter from the ratio of the emission coefficients. Actually, the observed emission lines always have an associated intrinsic noise. As a consequence, deconvolution and inversion processes are in gen- eral "ill-conditioned" problems (i.e. similar initial conditions lead to very different deconvolved/inverted solutions). The level of ill-conditioning depends on the particular form of the convolution/integration operator ("the kernel"), and on the level of noise in the data (e.g. Craig \& Brown 1986; Brown 1995).

The convolution also produces a loss of information, that again depends on the form of the kernel. In fact, the convolution process may be seen as a low-pass filter. All the structures with size smaller than the characteristic size of the kernel (the PSF/seeing) will not be reconstructed properly when deconvolving the image.

In addition, the determination of the physical parameters from the emission coefficients depends on several assumptions and uncertainties as, for instance, those associated with the ratios of the populations of the emitting species (Podio et al. 2006).

Finally, to solve the tomography problem, it is necessary to make some assumptions about the geometry of the problem. In some cases, in stellar jets, a reasonable hypothesis is to consider the jet as cylindrically symmetric. This hypothesis describes better the three dimensional structure of the jet than the commonly used assumption of homogeneity along the line of sight.

\subsection{Reconstruction of the Jet Structure Using Tomographic Techniques}

Let us consider an axisymmetric jet moving in the plane of the sky. In the following, we use a twodimensional Cartesian reference system centered on the star-disk system, and defined on the plane of the sky, such that the $x$ - and $y$-axes are perpendicular and parallel (respectively) to the "main" axis of the jet. Additionally, we indicate with $r$ the cylindrical distance from the jet axis.

The aim of the inversion process is to determine the emission coefficients $i(r, y)$ as a function of the observed intensities $I(x, y)$. The problem is further reduced to one dimension assuming that the variation of the physical parameters and therefore of the emission lines along the $y$-axis (parallel to the direction of propagation of the jets) is much smaller than the variation in the direction across the jet (i.e., along the $r, x$-axis).

With these approximations, the observed emission line intensity cross section $I(x)$ is related to the emission coefficient $i(r)$ by the Abel transform:

$$
I(x)=2 \int_{x}^{R} \frac{i(r) r d r}{\sqrt{r^{2}-x^{2}}},
$$

where $R$ is the jet radius. equation (1) represents an approximation of the relation between observed and volumetric emission coefficients (see Appendix A).

As mentioned previously, the inversion of equation (11) constitutes an ill-posed problem. The reason for that is easily seen looking at the analytical solution of the Abel transform, given by:

$$
i(r)=-\frac{1}{\pi} \int_{r}^{R} \frac{d I}{d x} \frac{d x}{\sqrt{x^{2}-r^{2}}} .
$$

Decomposing the data into a continuous noise-free and an oscillatory noisy part (expanded as a function of the frequencies $\left.\omega_{n}\right) I=I_{0}+\delta \sum_{n} \sin \omega_{n} x$, the derivative 
is: $d I / d x=d I_{0} / d x+\delta \sum_{n} \omega_{n} \sin \omega_{n} x$, and the high frequency noise dominates the integral of equation (2).

Equation (1) can be written as

$$
d=A x
$$

where $x$ is a vector of $M$ elements (a discretization of the solution $i(r)$ of the inverse problem), $d$ is a vector of $N$ elements representing the data $I\left(x_{i}\right)$, and $A$ is a matrix with dimension $M \times N$. A is an operator that transforms the volumetric into the integrated intensities, and is given by a particular discretization of the integral present in equation (11) (see Appendix A).

The least-squares solution of this linear system of equations is obtained minimizing the norm of the residuals:

$$
\min \|A x-d\|_{2}^{2},
$$

where the standard definition of the 2-norm of a vector of $n$ elements is given by

$$
\|x\|_{2}=\sqrt{x_{1}^{2}+x_{2}^{2}+\cdots+x_{n}^{2}} .
$$

For noisy data, the solution determined minimizing the norm of the residuals (eq. 4), as shown before, is dominated by high frequency noise. A standard solution to this problem (e.g., Craig \& Brown 1986; Aster et al. 2005) is obtained by "regularizing" the data, which in the Tikhonov approach consists of solving the following damped least squares problem:

$$
\min \left(\|A x-d\|_{2}^{2}+\alpha^{2}\|L x\|_{2}^{2}\right),
$$

with the condition:

$$
\|A x-d\| \leq \delta
$$

where $\alpha$ is the "regularization parameter", $L$ is the "Tikhonov matrix", and $\delta$ is the uncertainty (i.e., the noise level) in the data. $L$ can be chosen to minimize the norm of the solution, or the first or second derivatives of the solution (see Appendix A).

Basically, the Tikhonov method chooses the smoothest solution (with respect to the zero-, first-, second-, etc. order derivative, depending on the particular form of $L$ used) such that the residuals are of the order of the noise in the data. The value of $\alpha$ obtained by equation (7) determines the "optimal" (i.e. the smoothest) solution compatible with the data and their associated errors. In particular, while a too small value of $\alpha$ produces a solution dominated by noise, a larger value of $\alpha$ produces a too smooth solution. Finally, it is important to note that the solution of the inverse problem depends on the particular form of the Tikhonov matrix $L$ (that is a free parameter of the problem). This is related to the wellknown fact that, to solve an inverse problem, it is necessary to introduce a certain amount of information on the form of the solution (e.g., on the smoothness of the solution or its derivative of order $n$ ).

Once the emission coefficients have been reconstructed, the physical parameters are determined from their ratios.

\section{RESULTS}

\subsection{The HН30 Data}

The HH30 jet is one of the best studied outflows from T Tauri stars (Mundt et al. 1991; Burrows et al. 1996: Rav et al. 1996; Bacciotti et al. 1999; Pety et al. 2006;

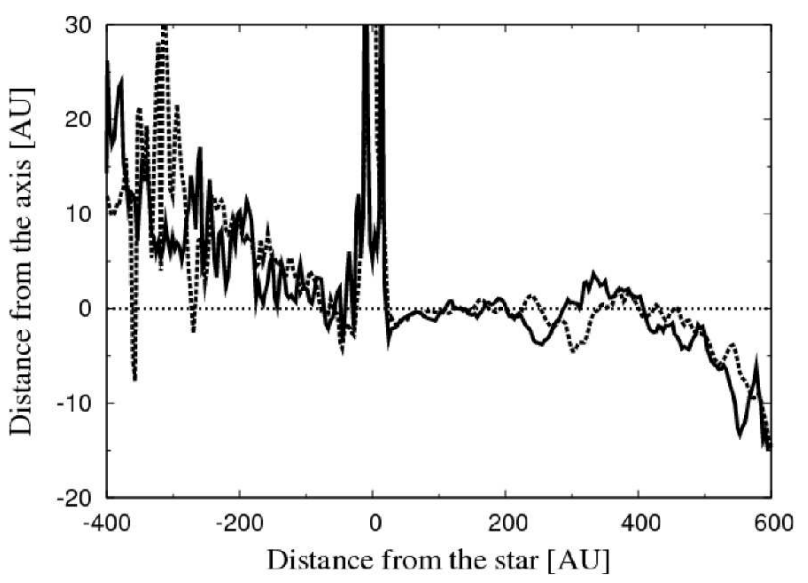

FIG. 1.- Position of the jet axis $x_{0}$ relative to the star. The $x$ and $y$-axis are parallel and perpendicular to the main axis of the jet, respectively. Negative/ positive $x$ distances correspond to the redshifted/blueshifted outflow.

Anglada et al. 2007, HM07). It is located at a distance of 140 pc (Kenyon et al. 1994), in the Taurus molecular cloud. HH30 is nearly on the plane of the sky, within an angle of $\sim 1^{\circ}$, and it is approximately axisymmetric. Because the outflow lies so close to the plane of the sky, the flared disk absorbs a large part of the radiation coming from the star, allowing the study of the jet at distances $\gtrsim 20$ AU from the star. The jet is bipolar, but with a blueshifted lobe much more luminous than the redshifted lobe.

Furthermore, high resolution, multi-epoch images of this jet are available. The data, presented by HM07, were obtained by the Space Telescope Imaging Spectrograph (STIS) on HST using a slitless spectroscopy technique, during two epochs, in 2000 and 2002. They consist of two-dimensional images of the jet in a series of emission lines, including four bright optical lines: the $[\mathrm{S}$ II $] \lambda \lambda 6716,6731$ doublet, the $[\mathrm{O} \mathrm{I}] \lambda 6300$ line and the [N II] $\lambda 6583$ line.

In the images, the cross section of the jet (i.e., in the direction perpendicular to the jet axis) is resolved with $\sim 15-20$ pixels, depending on the distance from the central star. As determined by HM07, the PSF FWHM is of $\sim 4$ pixels, with each pixel size corresponding to $0.025^{\prime \prime}$ or $3.55 \mathrm{AU} /$ pixel. As stated by $\mathrm{HM} 07$, the $\mathrm{S} / \mathrm{N}$ is very high along the jet knots, with uncertainties below $5 \%$.

\subsection{Line reconstruction}

The Tikhonov regularization technique has been applied to the inversion of the observed data, to reconstruct the emission coefficients.

To invert equation (1) it is necessary to know the position $x_{0}$ of the jet axis in the emission line images. As the HH30 jet is not perfectly axisymmetric, $x_{0}$ is a slowly varying function of $y$, i.e. $x_{0}=x_{0}(y)$. We determine $x_{0}(y)$ by a Gaussian fit to the total line intensity crosssection. The resulting $x_{0}$ is shown in Fig. 1.

The blueshifted jet bends at distances $\gtrsim 400$ AU, probably due to precession (Burrows et al. 1996), while the redshifted jet (much more noisy due to the lower $\mathrm{S} / \mathrm{N}$ 


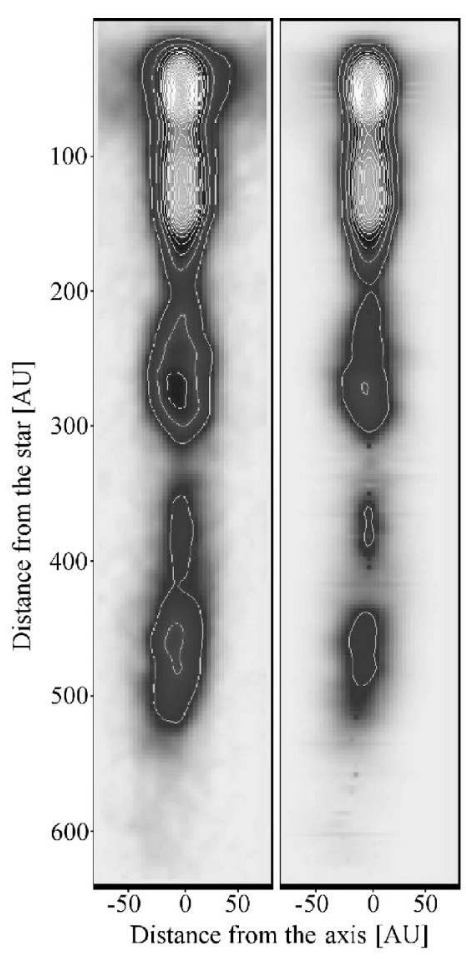

FIG. 2.- Observed (left) and reconstructed (right) emission line images (arbitrary units). The images refers to the sum of all emission lines (reconstructed independently). The central source is on the top of the Figure.

of the emission line images) it is not aligned with its blueshifted counterpart, forming an angle of $\approx 2.5^{\circ}$. It is unclear whether this asymmetry is due to inhomogeneities of the ambient medium or is a property of the ejection mechanism, although the fact that it starts close to the source seems to favor the latter explanation.

An asymmetry of the ejection mechanism itself could be related to an asymmetry in the stellar magnetic field. However, exploratory three-dimensional simulations by Romanova et al. (2009) of a stellar dipole magnetic field misaligned with the rotation axis of the star and the disk produce winds that are axisymmetric about the rotational axis. More complex magnetic field configurations have been studied by Lovelace et al. (2010). These authors used two-dimensional axisymmetric simulations to study the case of a rotating star with a large quadrupole component, showing that intrinsically asymmetric jets (with respect to the equatorial plane) can be generated in this case. Therefore, a possible origin for the asymmetry could be a large quadrupole component of the stellar magnetic field, misaligned with the star + disk rotation axis.

Consistently with the axisymmetry hypothesis, pixels symmetric with respect to $x_{0}$ are combined to produce symmetric emission-line cross-sections. The emission coefficients are then determined inverting the Abel transform (eq. 11) for each emission line. The resulting emission line images (Fig. 2) are sharper than the original, while still conserving the main features of the jet (e.g. the position and number of knots).

However, sharper emission lines do not necessarily imply different line ratios and therefore different physical
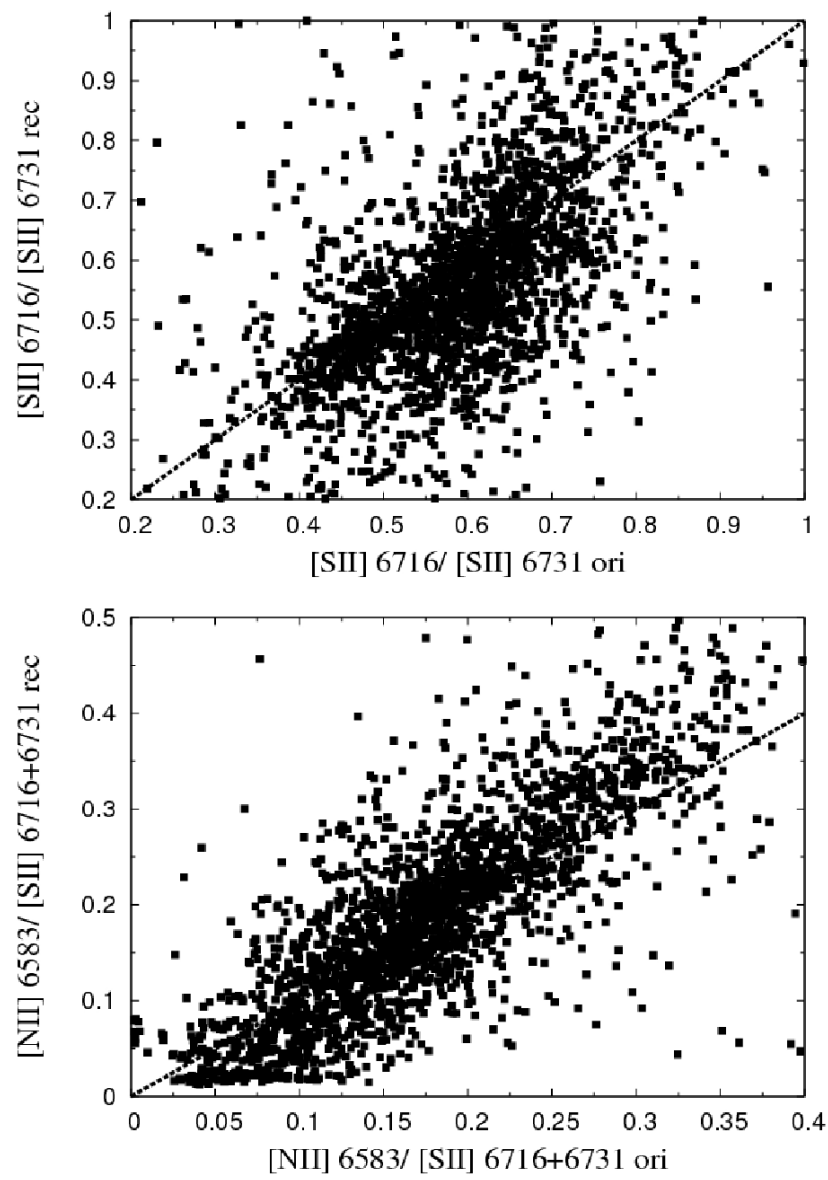

FIG. 3.- Reconstructed vs. original $[\mathrm{N}$ II]/[S II] ratio (upper panel) and [S II] 6716/[S II] 6731 ratio (lower panel). Only pixels with emission lines with $\mathrm{S} / \mathrm{N}>10$ are included. The dashed line shows the curve $y=x$.

parameters. In fact (see Paper I), in the case of an observed emission line with a gaussian dependence on $x$ : $I(x) \propto \exp \left(-(x / \sigma)^{2}\right)$, the emission coefficients scale as $i(r) \propto 1 / \sigma \exp \left(-(r / \sigma)^{2}\right)$. The relation between the reconstructed and original line ratios is therefore

$$
\frac{i_{1}}{i_{2}} \propto \frac{\sigma_{2}}{\sigma_{1}} \frac{I_{1}}{I_{2}} .
$$

The [S II] 6716/[S II] 6731 and [N $\mathrm{II}] 6583 /\left[\begin{array}{ll}\mathrm{S} & \mathrm{II}\end{array}\right]$ $6716+6731$ reconstructed ratios are shown in fig. 3 as a function of the observed ratios.

The [S II] 6716/[S II] 6731 reconstructed ratio is systematically lower than the original one, with a larger effect for lower values of the ratio. As this ratio is a monotonic decreasing function of $n_{\mathrm{e}}$, this result implies that the inversion process produces in general larger electron density, with a larger increase for densities close to the high density limit. The large spread in the data is not related to errors in the tomographic inversion process. The inversion of a pixel with a certain [S II] ratio is not unique, as it depends on the emission line cross-sections and not only on its own value.

The [N II] 6583/[S II] $6716+6731$ ratio presents a more complex behavior. It depends on all the physical parameters (with a stronger dependence on $x_{\mathrm{H}}$ ), and it is 


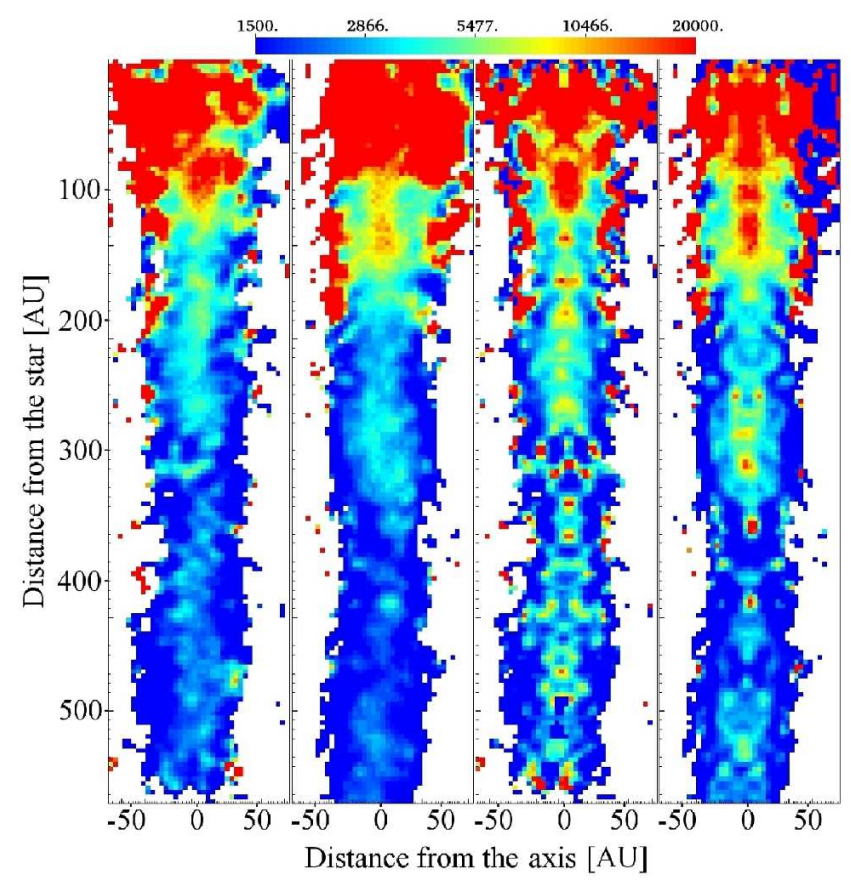

FIG. 4.- Electron density for the first and second epoch for the original (left panels) and reconstructed emission line images (right panels). The $\mathrm{x}$-axis represents the direction perpendicular to the jet axis (with a size of $60 \mathrm{AU}$ ), while the y-axis represents the direction parallel to the jet axis (500 AU). The source is located at the top of the Figure.

a good shock tracer (HM07), as it becomes higher for denser, hotter and more ionized gas. Fig. 3] show that this reconstructed ratio increases (with respect to the original) for high values, while slightly decreasing for low values, indicating that shocks will be more evident in the reconstructed jet.

\subsection{The Determination of the Physical Parameters}

To determine $n_{\mathrm{e}}, T_{\mathrm{e}}$ and $x_{\mathrm{H}}$ we use the BE method (Bacciotti \& Eislöffel 1999). In this method, Nitrogen and Oxygen ionization fractions are determined assuming charge exchange equilibrium with Hydrogen, while Sulfur is assumed to be completely ionized. From four emission lines, three independent ratios are formed. Being the ratios just function of $n_{\mathrm{e}}, T_{\mathrm{e}}$ and $x_{\mathrm{H}}$ (fixing the relative population of $\mathrm{S}, \mathrm{O}, \mathrm{N})$, these parameters can be determined easily by any root-finding algorithm.

This procedure has been improved recently by HM07, including all the available ratios. HM07 showed that the best estimation of the physical parameters is the one obtained by the minimization of the least square problem

$$
\min \sum_{k=1}^{p} \frac{\left(r_{k}-m_{k}\right)^{2}}{\sigma_{k}^{2}}
$$

where $m_{k}=m_{k}\left(n_{\mathrm{e}}, T_{\mathrm{e}}, x_{\mathrm{H}}\right)$ is the model prediction, $r_{k}$ is the natural logarithm of the observed $k$-ratio, $p$ is the total number of ratios considered, and $\sigma_{k}$ is the error in the $k$-observed ratio.

The electron density, temperature and ionization fraction obtained from the observed and inverted ratios are shown in fig. 4, 6 for the two epochs, while the derived total hydrogen density is shown in fig. 7.

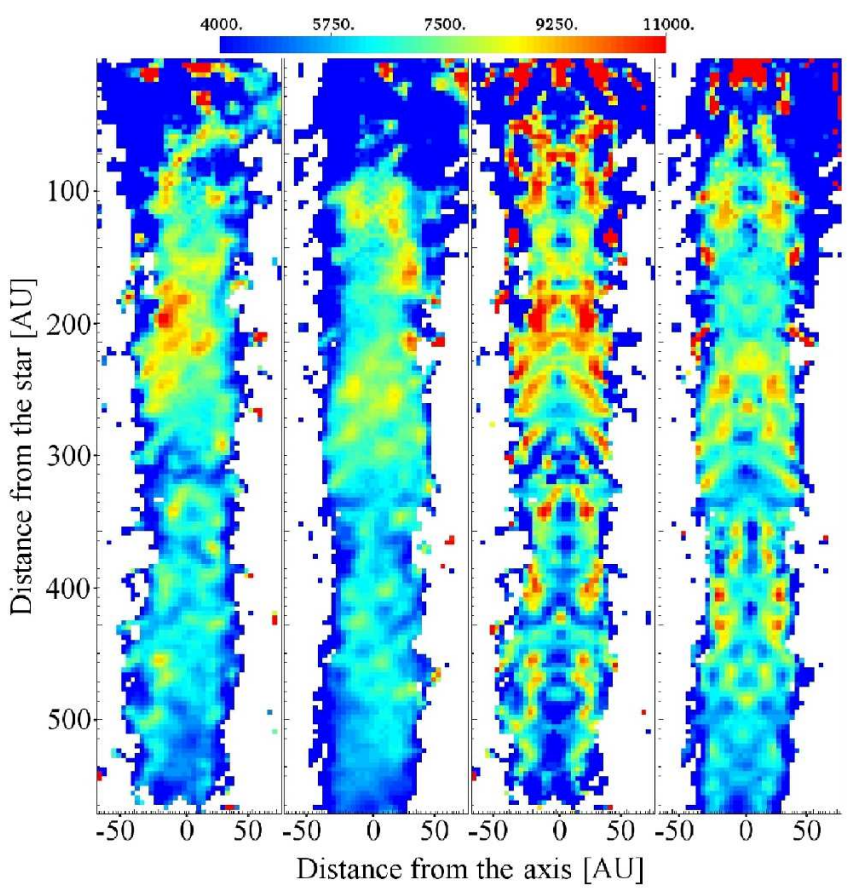

FIG. 5.- The same as in Fig. 4 but for the electron temperature.

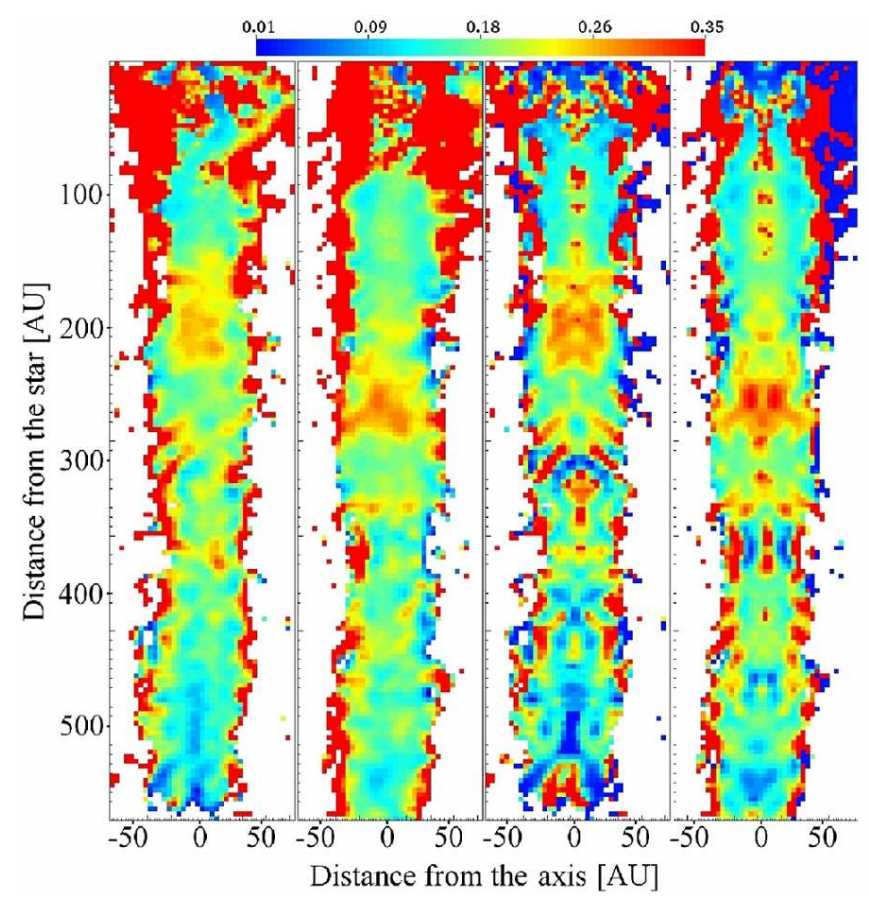

FIG. 6. - The same as in Fig. 4 but for the ionization fraction.

In general, the reconstructed $n_{\mathrm{e}}, T$ and $x_{\mathrm{H}}$ cross sections are much steeper than the observed cross sections of the same parameters. The [S II] ratio is in the high density limit close to the source, and the electron densities in that region are $\gtrsim 2 \times 10^{4} \mathrm{~cm}^{-3}$. This region can be identified as the saturated region in Fig. 4. While both the observed and the reconstructed electron densities drop with the distance from the source, the detailed behavior is quite different. In fact, the reconstructed electron 


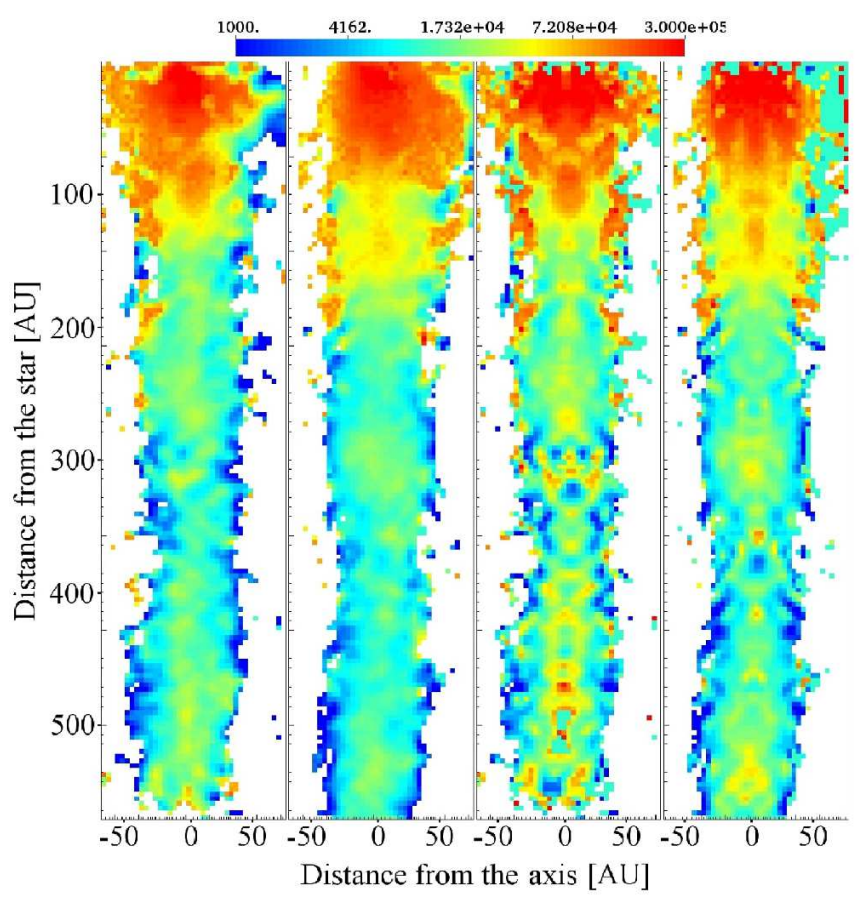

FIG. 7.- The same as in Fig. 4 but for the hydrogen density $\left(n_{\mathrm{H}} / n_{\mathrm{e}}\right)$.

density presents a series of stronger on-axis peaks with $n_{\mathrm{e}} \gtrsim 10^{4} \mathrm{~cm}^{-3}$ extending along all the jet. In those regions, the ionization fraction also increases, while the temperature tends to present on-axis valleys. Corresponding to lower values of $T_{\mathrm{e}}$ on the axis, the jet presents regions of $T_{\mathrm{e}} \gtrsim 10^{4} \mathrm{~K}$ at $\approx 20 \mathrm{AU}$ from the jet axis. The inversion process produces total densities $\left(n_{\mathrm{H}}=n_{\mathrm{e}} / x_{\mathrm{H}}\right)$ that are also larger on the jet axis with respect to the jet edge, although this difference is lower than the corresponding difference in the electron density. While the $n_{\mathrm{e}}$ increases by a factor of $\sim 2$ (as in HM07) from the edge to the axis of the jet for the observed data, the reconstructed $n_{\mathrm{e}}$ increases by a larger factor, of order of $\sim 5-10$ depending on the position along the jet. In general, high electron density regions are more strongly affected by the reconstruction process than low density regions. This is direct consequence of the stronger dependence on density of the $[\mathrm{S} \mathrm{II}]$ ratio close to the high-density limit (see also Fig. 8 of Paper I).

The ionization fraction (both observed and reconstructed) seems to increase on the edge of the jet up to values $\gtrsim 0.5$. On the edge of the jet, the $\mathrm{S} / \mathrm{N}$ becomes low and the error in the determination of the physical parameters becomes dominant, so this result is probably affected by numerical uncertainties. Interestingly, the increase in the ionization fraction on the edges of the jet is a result expected in shock models, as pressure gradients in the post-shock region move the ionized gas laterally in the cocoon of the jet, where a region of low density, high ionization fraction is created. The ionized gas present in that region recombines on a timescale much larger than on the axis of the jet, where the density is orders of magnitude larger.

\section{4. $F W H M$}

The degree of collimation of the jet can be estimated by the FWHM. Typical T-Tauri star jets, including HH30, have an opening angle of a few degrees at $\sim 100 \mathrm{AU}$ from the source, consistent with magnetic self-collimation models (Rav et al. 2007). HM07 measured a deconvolved FWHM of $\approx 10$ AU close to the HH30 source, with an opening angle of $2.6^{\circ}$. HM07 also showed that there is no clear correlation between the jet collimation/width and the presence of a knot.

We have calculated the FWHM before and after the inversion (Fig. 8) for the sum of the [S II], [O I] and [N II] emission lines. We have also deconvolved the FWHM determined from the original and the reconstructed data, assuming a FWHM PSF of 4 pixels (HM07). The deconvolution of the reconstructed data gives just an approximated estimate of the real FWHM, as the deconvolution should be applied to the data before the reconstruction is made and not after (see eq. A1).

Fig. 8 shows that results similar to HM07 are obtained for the FWHM calculated from the observed and the deconvolved images. Also, Fig. 8 shows that the inversion and the deconvolution produce a comparable effect on the FWHM, which has a similar amplitude at small distances from the source, going from a width of $\sim 15 \mathrm{AU}$ at $20 \mathrm{AU}$ to $30 \mathrm{AU}$ at a distance of $500 \mathrm{AU}$ from the source. At larger distances the FWHM computed from the image inverted by tomography is slightly lower than that obtained from the deconvolved image. The value of the FWHM determined by deconvolving the reconstructed image is much smaller, going for instance from $5 \mathrm{AU}$ at $30 \mathrm{AU}$ to $25 \mathrm{AU}$ at $500 \mathrm{AU}$ for the first epoch. The opening angle is $2.4^{\circ}$, similar to the one determined by HM07.

The reconstruction process also shows some correlation between the knots and the width of the jet. In fact, the first two knots in the first epoch and the first knot in the second epoch (fig. 8) clearly correspond to a drop in the FWHM, while the rest of the knots do not present any correlation. That seems to imply that the knot material is actually ejected more collimated from the star-disk system than the material outside the knots. The reason for the loss of correlation seems therefore to be related to the presence of collisions between knots.

\subsection{Mass Loss}

Assuming a uniform velocity cross-section of $200 \mathrm{~km}$ $\mathrm{s}^{-1}$, and using the density stratification coming from the inversion process, we have determined the mass-flux by integrating $d \dot{M} \propto$ rvndr. The resulting mass-fluxes computed from the original and the reconstructed densities are similar. They rapidly drop from $10^{-8} \mathrm{M}_{\odot} \mathrm{yr}^{-1}$ close to the source to $10^{-7} \mathrm{M}_{\odot} \mathrm{yr}^{-1}$ at $200 \mathrm{AU}$, that are typical values for jets from T-Tauri stars (e.g., Hartigan et al. 1995, Lavallev-Fouquet et al. 2000). Interestingly, a very different result is obtained assuming a power-law profile for the velocity. If, for instance, $v \propto r^{-\eta}$, values of $\eta \gtrsim 1$ gives mass fluxes $\lesssim 10^{-8} \mathrm{M}_{\odot} \mathrm{yr}^{-1}$ close to the source, and approximately one order of magnitude lower at at distance of $\gtrsim 200 \mathrm{AU}$ from the source. These values of the mass-flux are much less than those obtained by using other methods (e.g., Hartigan et al. 1995, Lavalley-Fouquet et al. 2000), implying apparently that the velocity cross-section has to be nearly flat (i.e. with 

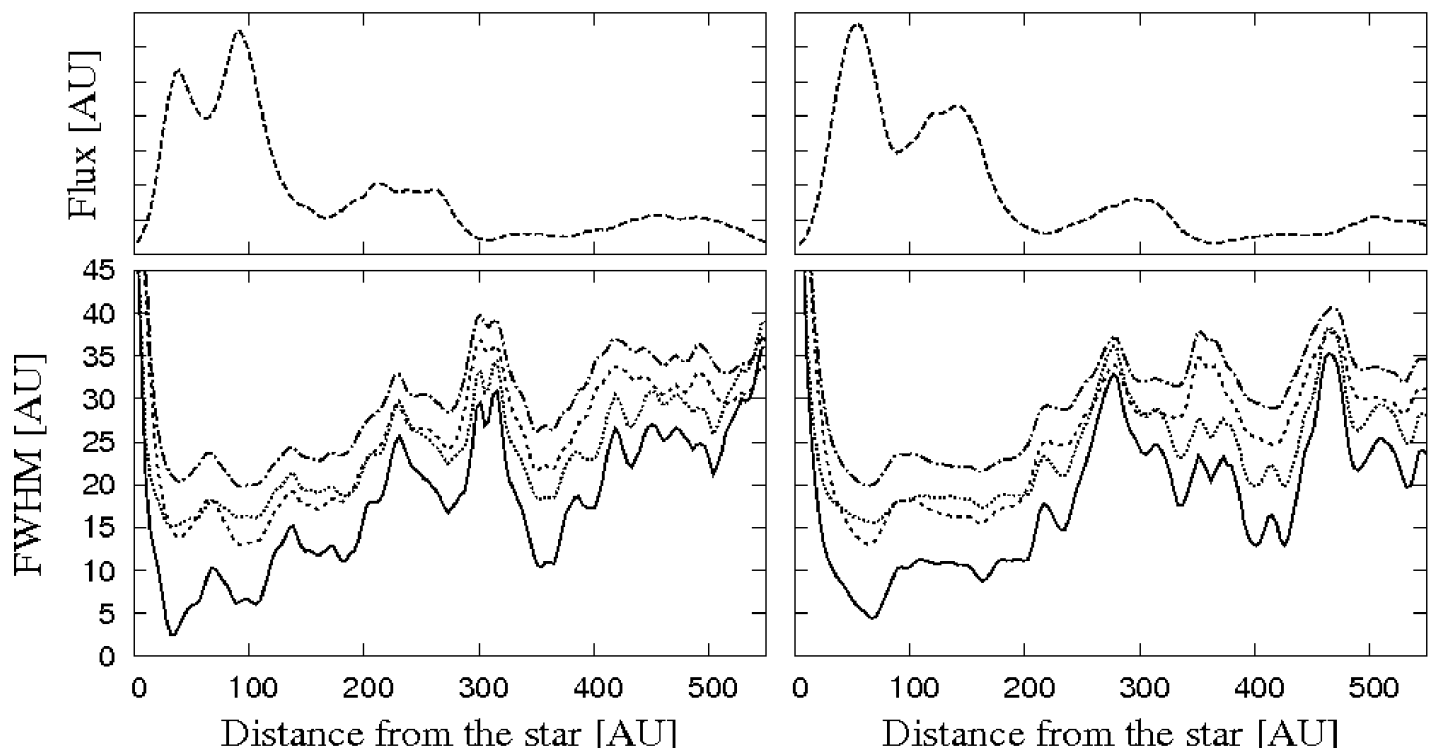

FIG. 8.- Upper panels: Emission line flux (in arbitrary units) as a function of the distance from the star, for the I (left) and II epoch (right). The flux is obtained adding the [S II], [N II] and [O I]. Lower panels: FWHM as a function of distance from the star, for the integrated emission line intensities (shown in the upper panels of the figure). The different curves correspond to the observed (dash-dotted line), reconstructed (dotted), deconvolved (dashed) and reconstructed and deconvolved (continuous) data. The deconvolution has been done assuming a FWHM PSF of 4 pixels.

a velocity gradient with $\eta \lesssim 0.5)$ across the jet crosssection. Alternatively, these results can imply that the on-axis total density value is much larger than the one derived by the reconstruction process.

\subsection{Errors}

There are three main sources of error that affect the results of this work: errors due to the hypothesis of axisymmetery, errors related to the assumptions of the $\mathrm{BE}$ method, and errors due to the PSF convolution. They are discussed in some detail in the following.

The first source of errors is related to the hypothesis of axisymmetry. To understand how asymmetries present in the HH30 jet affect our results, we have determined the physical parameters for both sides of the jet independently (see 3.2 ). The physical parameters obtained differ from those shown by less than 30\%, without any systematic effect present.

The second source of errors is related to the approximations used in the BE method. In particular, the main errors in the BE method are related to the uncertainty in the elemental abundances. Podio et al. (2006) have shown that the choice of elemental abundances affects the derivation of the physical parameters up to $40 \%$ for $x_{e}$ and $25 \%$ for $T_{\mathrm{e}}$. On the other side, the determination of $n_{\mathrm{e}}$, coming from the ratio of sulfur lines, is not affected by the choice of the relative abundances. Furthermore, the errors in the determination of the electron density are larger close to the star, where the [S II] 6716/6731 ratio is in the high density limit.

The third source of errors is related to the PSF. In this work we have not deconvolved the data with the PSF, for two reasons. First, the exact form for the PSF is not available. Second, and more important, the mathematical problem becomes much more complex if the PSF deconvolution is included (eq. A1). As we have seen in the determination of the FWHM, the effect of the PSF is of the same order as the effect produced by the inversion process itself close to the source, and is of lower importance at larger distance from the source. Anyway, the inversion process itself is limited by the presence of noise in the data. A large level of noise implies the need to use a larger regularization parameter. In other words, the presence of a certain level of noise implies a loss of information, that cannot be recovered in the inversion process. However, the PSF deconvolution would produce effects in the same direction as the inversion process, resulting in steeper profiles, with larger densities and ionization fraction, and lower temperatures on-axis. The results of this paper remain still valid at least qualitatively, and represent a huge improvements with respect to previous works in which neither the PSF deconvolution nor the line-of-sight integration were considered in the determination of the physical parameters.

\section{DISCUSSION}

The main results of this paper are that the inverted electron density is in general larger, and that it presents a series of sub-structures not clearly evident in the $n_{\mathrm{e}}$ determined directly from the observed data. The same effect is evident in the total density, although in lower measure, as the increase in electron density corresponds to a (lower) increase in the ionization fraction.

This result has important implications for our understanding of the origin of $\mathrm{HH}$ objects. The presence of knots in stellar jets seems to be related to the generation of internal working surfaces due to supersonic variations of the ejection velocity at the base of the jet (Raga et al. 1990). On the other side, the morphology of the HH30 jet is not easily modeled in terms of variable hydrodynamics jet models, because a periodic velocity ejection from the central source is more likely to produce knots with "bow-shock-like" morphology (e.g., as seen in HH 111, Reipurth et al. 1996). A possible explanation has been proposed by De Colle \& Raga (2006), who showed that axially elongated structures such as the ones observed in the HH30 jet can be produced by pinch instability driven by strong toroidal magnetic fields (possibly co- 
existing with the presence of internal working surfaces). The results of this paper suggest a possible alternative explanation. In fact, the presence of sub-structures in the elongated knots indicate that a velocity variation with lower timescales, maybe of chaotic nature, can be operating, superimposed to the large scale velocity variation responsible for the creation of knots. The scenario of chaotic ejection of material forming the finally visible jet knots has recently been explored in numerical simulations by Yirak et al. (2009) and Bonito et al. (2010).

The reconstructed jet presents regions of lower temperature and higher ionization fraction. In particular, lower temperature on-axis regions are qualitatively consistent with shock models, due to the stronger cooling that one would expect in higher density regions.

The same technique could in principle be applied to other existing data, although other jets have in general inclination angles different from zero and, as a consequence, the interpretation of the results would be more complex (e. g. Bacciotti et al. 2000, 2002; Coffey et al. 2004; Woitas et al. 2005; Coffey et al. 2007; Beck et al. 2007, HM07).

The HH30 data presented in HM07 have a very high $\mathrm{S} / \mathrm{N}$ level. While the same technique can in principle be applied to data with higher uncertainties, our ability to understand the structure of the jet is limited by the noise level of the observations, as the regularization techniques give smoother solutions for data with lower $\mathrm{S} / \mathrm{N}$.

Observations obtained with integral field spectroscopes (i.e. three-dimensional spectral data with twodimensional spatial coverage), in particular, can be used to extract information also on the dependence of the velocity on $r$. In this case, in fact, the relation between the observed intensity and the emission coefficient is given by

$$
I(x, v)=2 \int_{x}^{\infty} \frac{i(r, v) r d r}{\sqrt{r^{2}-x^{2}}}
$$

with

$$
i(r, v)=\frac{i(r)}{\sqrt{\pi} \sigma_{v}} e^{-(v-v(r))^{2} / \sigma_{v}^{2}}
$$

where $\sigma_{v}$ is the velocity dispersion. Given the observed line intensity $I(x, v), i(r, v)$ can be determined from equation (10), $i(r)$ can be determined by inverting the Abel equation (related to eq. [1] by $I(x)=\int I(x, v) d v$ ) and $v(r)$ can be finally inferred from equation (11). In practice, $\sigma_{v}$ is often dominated by the instrumental PSF (e.g. $10-20 \mathrm{~km} \mathrm{~s}^{-1}$ vs. a thermal width of $\sim$ a few $\mathrm{km} \mathrm{s}^{-1}$ for ions as S II or O I). On the other side, often $v \gg \sigma_{v}\left(\sim 100 \mathrm{~km} \mathrm{~s}^{-1}\right.$ both across and along the jet $)$ and important information about the dynamics of the jet can be determined from $v(r)$, as for instance the mass-flux.

In cases when information on the width of the jet in two different lines (i.e. the [S II] doublet) is available, equation (8) can be used (see also eq. [18] of Paper I) to have an order of magnitude estimation of the effect of projection effects.

\section{CONCLUSIONS}

In this paper we have for the first time applied the tomographic inversion technique to determine, under the hypothesis of axisymmetry, the three dimensional structure of the HH30 jet. This hypothesis describes better the three dimensional structure of the jet than the commonly used assumption of homogeneity along the line of sight.

The main results are summarized as follows: the reconstructed electron and total densities show a fragmented structure, probably consistent with small timescale $(\sim$ months) ejection velocity variations. Corresponding to the peaks in electron and hydrogen density, the ionization fraction also increases, while the electron temperature presents on-axis valleys. The width of the reconstructed jet is lower than the corresponding width inferred directly from the observations. In particular, projection and instrumental effects have a similar effect on the FWHM. Combining the two effects, the FWHM becomes $\lesssim 10$ AU close to the source. Although our determination of the mass-flux is limited by the lack of information on the velocity radial stratification, we have shown that the effect of the density stratification on the mass-flux evaluation is negligible.

The application of the BE technique to the HH30 jet is limited to distances from the source $\gtrsim 40 \mathrm{AU}$, where the [S II] 6716/6731 ratio is not in the high density limit, and is possible to properly determine the electron density.

Future observations dedicated to the study of the jet close to the source, using line ratios with larger critical densities, may allow a direct comparison between the results of the inversion and predictions of jet ejection models. In this way, it will be possible to compare for example the density profiles obtained from the inversion with theoretical predictions. For instance, the disk-wind and $\mathrm{X}$-wind predict different power-law dependencies of the density as a function of the distance from the jet axis, while the magnetic tower model predicts a sharp outer jet edge (e.g. Shu et al. 1995; Lery \& Frank 2000).

We have shown that tomographic reconstruction can be used to reconstruct the cross section of the flow, obtaining a description of the three dimensional structure of the jet. The use of high resolution observations will make it possible to apply this technique to a larger sample of objects.

We thank Pat Hartigan for sharing with us his HSTHH30 data.

\section{APPENDIX}

\section{IMPLEMENTATION OF THE TIKHONOV REGULARIZATION}

Here we present the details of our implementation. For a more general description of the Tikhonov regularization technique see for instance Aster et al. (2005); Craig \& Brown (1986).

The observed intensity $I\left(x_{i}, y_{j}\right)$ is related to the emission coefficients $i(r, y)$ by the following expression:

$$
I\left(x_{i}, y_{j}\right)=2 \int_{x_{i-1 / 2}}^{x_{i+1 / 2}} d x \int_{y_{j-1 / 2}}^{y_{j+1 / 2}} d y \int_{-\infty}^{+\infty} \int_{-\infty}^{+\infty} \psi\left(x-x^{\prime}, y-y^{\prime}\right) d x^{\prime} d y^{\prime} \int_{x^{\prime}-x_{0}(y)}^{R} \frac{i(r, y) r d r}{\sqrt{r^{2}-\left(x^{\prime}-x_{0}(y)\right)^{2}}}
$$


The first two integrals of equation (A1) represent the integration on the pixel centered on $\left(x_{i}, y_{j}\right)$ with dimension $\left(x_{i-1 / 2}, x_{i+1 / 2}\right) \times\left(y_{j-1 / 2}, y_{j+1 / 2}\right)$. The third and fourth integrals are the convolution with the seeing and the instrumental response $\psi$, and the last term represents the integration along the line of sight, being $x_{0}(y)$ the position of the center of the jet (in general a function of the $y$ coordinate).

To simplify the problem, we neglect the effect of the instrumental response, assuming $\psi\left(x-x^{\prime}, y-y^{\prime}\right) \approx \delta\left(x-x^{\prime}, y-y^{\prime}\right)$, being $\delta(x)$ the delta function. Furthermore, we reduce the problem to one dimension assuming $i(r, y) \approx i\left(r, y_{j}\right)$, i.e. that the variations in the emission coefficients along $y$ are much smaller than those along $r$.

With these approximations, equation (A1) reduces to

$$
I\left(x_{i}, y_{j}\right)=2 \Delta y_{j} \int_{x_{i-1 / 2}}^{x_{i+1 / 2}} d x \int_{x-x_{0}\left(y_{j}\right)}^{R} \frac{i\left(r, y_{j}\right) r d r}{\sqrt{r^{2}-\left(x-x_{0}\left(y_{j}\right)\right)^{2}}},
$$

where $\delta y_{j}=y_{j+1 / 2}-y_{j-1 / 2}$. We further assume $\Delta y_{j}=1$ (the exact value of $\Delta y_{j}$ is not important, as it cancels out in the line ratios), and we indicate $I\left(x_{i}, y_{j}\right)=I_{i}$ and $i\left(r, y_{j}\right)=i(r)$.

As shown in 2.2 equation (A2) is inverted using the Tikhonov regularization technique, i.e. solving the following damped least square problem

$$
\min \left(\|A x-d\|_{2}^{2}+\alpha^{2}\|L x\|_{2}^{2}\right)
$$

with the condition

$$
\|A x-d\| \leq \delta
$$

In matrix form, equation (A3) can be written as

$$
\left(A^{T} A+\alpha^{2} L\right) x=A^{T} d
$$

where $A^{T}$ is the transpose matrix of $A$. The solution is obtained inverting this linear system of equations.

As discussed in 92.2 , the matrix $L$ can be chosen to minimize the norm of the solution, or its first or second derivative. In the first case (zero-order) solutions with low magnitude of $x$ are favored, while in the cases of first- and second- order regularization, the magnitude of the gradient and Laplacian are penalized, respectively. Being the solution unknown, the choice of the particular form of $L$ is somehow arbitrarily. In this work, we decide to minimize the first derivative of the solution as, in our implementation, it produces a less noisy solutions with respect to zero- and second-order minimization, and it works better in our tests. The matrix $L$ is therefore given by:

$$
L=\left|\begin{array}{ccccc}
-1 & 1 & \ldots & 0 & 0 \\
0 & -1 & \ldots & 0 & 0 \\
\ldots & \ldots & \ldots & \ldots & \ldots \\
0 & 0 & \ldots & -1 & 1 \\
0 & 0 & \ldots & 0 & -1
\end{array}\right|
$$

To complete the description of the algorithm, we need to specify the form of the matrix $A$. Let us assume that we have $N$ values of $I_{k}$, defined in the positions $x_{k}$ (with $0 \leq k \leq N-1$, where $x_{0}=\Delta x_{0} / 2$ ). The external radius is defined as $x_{N+1 / 2}=R$. Equation (A2) can be therefore written as:

$$
I_{k}=2 \int_{x_{k-1 / 2}}^{x_{k+1 / 2}} d x\left[\int_{x}^{x_{k+1 / 2}} \frac{i_{k}(r) r d r}{\sqrt{r^{2}-x^{2}}}+\sum_{j=k+1}^{N} \int_{x_{j-1 / 2}}^{x_{j+1 / 2}} \frac{i_{j}(r) r d r}{\sqrt{r^{2}-x^{2}}}\right]
$$

Taking $i_{k}(r)=i_{0, k}$ constant inside each shell, this equation leads to:

$$
I_{k}=i_{0, k}\left(S_{k+1 / 2}^{k+1 / 2}-S_{k-1 / 2}^{k+1 / 2}\right)+\sum_{j=k+1}^{N} i_{0, j}\left(S_{k+1 / 2}^{j+1 / 2}-S_{k+1 / 2}^{j-1 / 2}-S_{k-1 / 2}^{j+1 / 2}+S_{k-1 / 2}^{j-1 / 2}\right)
$$

where $S_{m}^{n}=x_{m} \sqrt{x_{n}^{2}-x_{m}^{2}}+x_{n}^{2} \arcsin x_{m} / x_{n}$, and the following relation has been used: $2 \int \sqrt{x_{n}^{2}-x^{2}} d x=$ $x \sqrt{x_{n}^{2}-x^{2}}+x_{n}^{2} \arcsin x / x_{n}=S^{n}$. The matrix $A$ can be easily deduced from equation (A7).

We have run a series of tests to verify the accuracy of the algorithms developed in this paper. Fig. 9 shows one of these tests. Assuming a certain radial dependence for electron density, temperature and ionization fraction, we have computed synthetic emission line intensities. We have then integrated the volumetric emission line along the line of sight using equation (A2), and then added a Poisson noise to the data. Finally, we have reconstructed the electron density, temperature and ionization fraction using the tomographic technique and the BE method ( 3.3 ). As shown in Fig. 9, the resulting physical parameters agree with the original one within 10\%. For comparison, we show in Fig. 9 also the physical parameter cross-sections obtained assuming the medium as homogeneous along the line of sight (i.e., those obtained from the "observed" emission lines). The tests show that also with a $10 \%$ noise level in the data our implementation of the tomographic inversion gives acceptable results.

\section{REFERENCES}

Anglada, G., López, R., Estalella, R., Masegosa, J., Riera, A., \& Raga, A. C. 2007, AJ, 133, 2799
Aster, E. C., Borchers, B., \& Clifford, C. H. 2005, Parameter Estimation and Inverse Problems (International Geophysic Series, vol. 90, Elsevier Academic Press, 2005, 301 p.) 


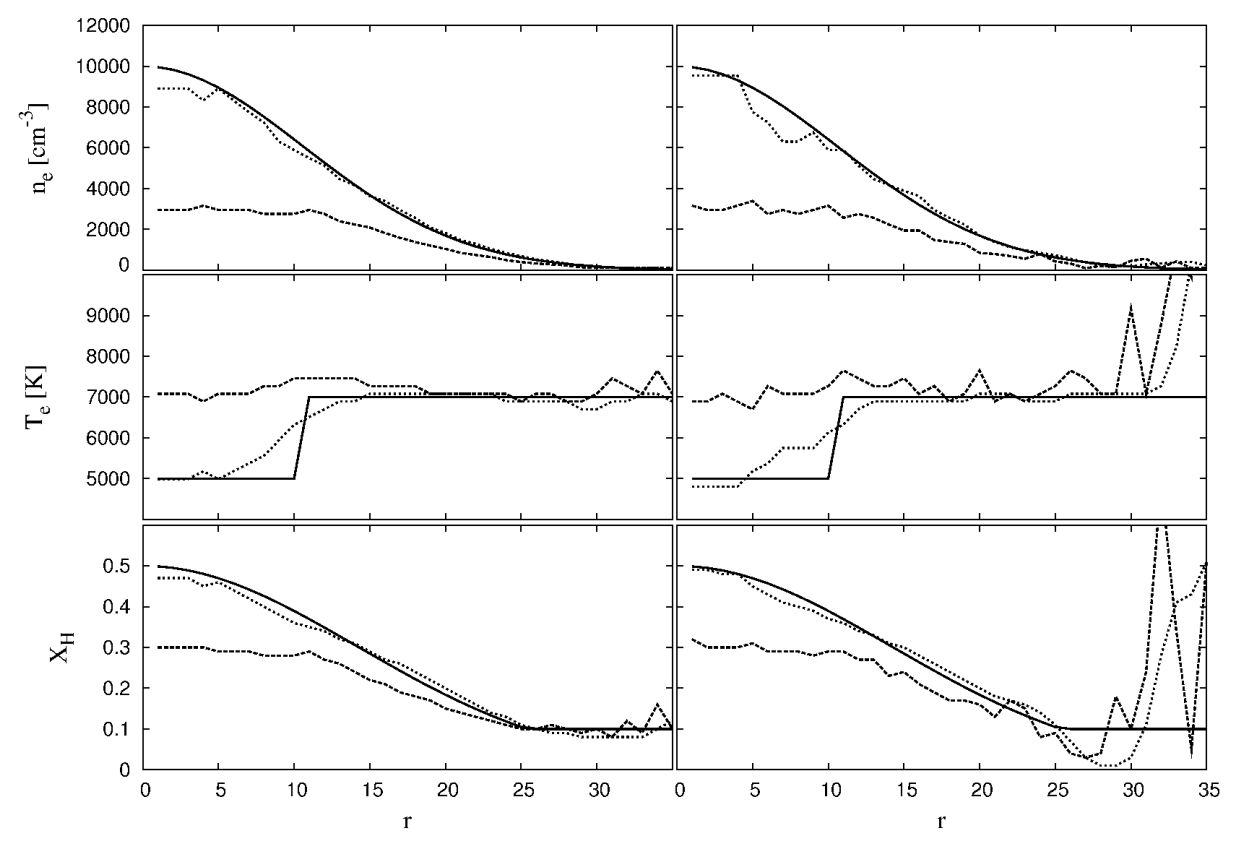

FIG. 9. - Test of the Tikhonov reconstruction technique for a Poisson noise with a variance of $5 \%$ (left column) and $10 \%$ (right column) at $r=5$. The different panels correspond to $n_{\mathrm{e}}$ (upper panels), $T_{\mathrm{e}}$ (center), $x_{\mathrm{H}}$ (lower) respectively. In each panel, the different curves represent the original profile (continuous line), that obtained from the integrated intensities (dashed line), and the reconstructed profile (dotted line). The reconstructed profiles is less steep than the original. The step in temperature is not well reconstructed by the Tikhonov technique. The edge of the jet is dominated by noise. The noise level of the physical parameters obtained from the integrated and reconstructed profiles are similar.

Bacciotti, F., \& Eislöffel, J. 1999, A\&A, 342, 717

Bacciotti, F., Eislöffel, J., \& Ray, T. P. 1999, A\&A, 350, 917

Bacciotti, F., Mundt, R., Ray, T. P., Eislöffel, J., Solf, J., \&

Camezind, M. 2000, ApJ, 537, L49

Bacciotti, F., Ray, T. P., Mundt, R., Eislöffel, J., \& Solf, J. 2002, ApJ, 576, 222

Beck, T. L., Riera, A., Raga, A. C., \& Reipurth, B. 2007, AJ, 133,1221

Bendinelli, O. 1991, ApJ, 366, 599

Bonito, R., Orlando, S., Peres, G., Eislöffel, J., Miceli, M. \& Favata, F. 2010, A\&A, 511, 42

Brown, J. C. 1995, Inverse Problems, 11, 783

Burrows, C. J., et al. 1996, ApJ, 473, 437

Carroll, J. J., Frank, A., Blackman, E. G., Cunningham, A. J., \& Quillen, A. C. 2009, ApJ, 695, 1376

Coffey, D., Bacciotti, F., Ray, T. P., Eislöffel, J., \& Woitas, J. 2007, ApJ, 663, 350

Coffey, D., Bacciotti, F., Woitas, J., Ray, T. P., \& Eislöffel, J. 2004, ApJ, 604, 758

Craig, I. J. D., \& Brown, J. C. 1986, Inverse problems in astronomy: A guide to inversion strategies for remotely sensed data (Research supported by SERC. Bristol, England and Boston, MA, Adam Hilger, Ltd., 1986, 159 p.)

De Colle, F., Del Burgo, C., \& Raga, A. C. 2008a, A\&A, 485, 765

De Colle, F., Gracia, J., \& Murphy, G. 2008b, ApJ, 688, 1137

De Colle, F., \& Raga, A. C. 2006, A\&A, 449, 1061

Dougados, C., Bacciotti, F., Cabrit, S., \& Nisini, B. 2010, in Lecture Notes in Physics, Berlin Springer Verlag, Vol. 793 , Lecture Notes in Physics, Berlin Springer Verlag, ed. P. J. V. Garcia \& J. M. Ferreira, 213-+

Ferreira, J. 1997, A\&A, 319, 340

Hartigan, P., Edwards, S., \& Ghandour, L. 1995, ApJ, 452, 736

Hartigan, P., \& Morse, J. 2007, ApJ, 660, 426

Hartigan, P., Morse, J. A., \& Raymond, J. 1994, ApJ, 436, 125

Hartigan, P., Raymond, J., \& Hartmann, L. 1987, ApJ, 316, 323

Hartmann, L. 2009, The Star-Jet-Disk System and Angular

Momentum Transfer, ed. Tsinganos, K., Ray, T., \& Stute, M., $23-32$
Kenyon, S. J., Dobrzycka, D., \& Hartmann, L. 1994, AJ, 108, 1872

Lavalley-Fouquet, C., Cabrit, S., \& Dougados, C. 2000, A\&A, $356, \mathrm{~L} 41$

Lery, T., \& Frank, A. 2000, ApJ, 533, 897

Lovelace, R. V. E., Romanova, M. M., Ustyugova, G. V., \& Koldoba, A. V. 2010, ArXiv e-prints

Mundt, R., Ray, T. P., \& Raga, A. C. 1991, A\&A, 252, 740

Nisini, B., Bacciotti, F., Giannini, T., Massi, F., Eislöffel, J., Podio, L., \& Ray, T. P. 2005, A\&A, 441, 159

Pesenti, N., Dougados, C., Cabrit, S., O'Brien, D., Garcia, P., \& Ferreira, J. 2003, A\&A, 410, 155

Pety, J., Gueth, F., Guilloteau, S., \& Dutrey, A. 2006, A\&A, 458, 841

Podio, L., Bacciotti, F., Nisini, B., Eislöffel, J., Massi, F., Giannini, T., \& Ray, T. P. 2006, A\&A, 456, 189

Raga, A. C., Binette, L., Canto, J., \& Calvet, N. 1990, ApJ, 364, 601

Raga, A. C., De Colle, F., Kajdič, P., Esquivel, A., \& Cantó, J. 2007, A\&A, 465, 879

Ray, T., Dougados, C., Bacciotti, F., Eislöffel, J., \& Chrysostomou, A. 2007, Protostars and Planets V, 231

Ray, T. P., Mundt, R., Dyson, J. E., Falle, S. A. E. G., \& Raga, A. C. 1996, ApJ, 468, L103+

Reipurth, B., \& Bally, J. 2001, ARA\&A, 39, 403

Reipurth, B., Raga, A. C., \& Heathcote, S. 1996, A\&A, 311, 989

Romanova, M. M., Ustyugova, G. V., Koldoba, A. V., \& Lovelace, R. V. E. 2009, MNRAS, 399, 1802

Shu, F. H., Najita, J., Ostriker, E. C., \& Shang, H. 1995, ApJ, $455, \mathrm{~L} 155+$

Shu, F. H., Najita, J. R., Shang, H., \& Li, Z. 2000, Protostars and Planets IV, 789

Woitas, J., Bacciotti, F., Ray, T. P., Marconi, A., Coffey, D., \& Eislöffel, J. 2005, A\&A, 432, 149

Yirak, K., Frank, A., Cunningham, A. J. \& Mitran, S. 2009 ApJ, 695,999 